\title{
52. FELDSPAR COMPOSITIONS OF VOLCANIC FLOW ROCKS FROM HESS RISE, DEEP SEA DRILLING PROJECT LEG 62 ${ }^{1}$
}

\author{
Florence Lee-Wong, U.S. Geological Survey, 345 Middlefield Road, Menlo Park, California
}

\begin{abstract}
Labradorite is the principal feldspar in tholeitic basalt from Deep Sea Drilling Project Site 464, on northern Hess Rise. Andesine and oligoclase constitute most primary feldspar in the reportedly trachytic rocks from southern Hess Rise at Site 465 . Secondary sanidine(?) has replaced the primary phases at Site 465 . The secondary potassium feldspar probably resulted from reaction of trachyte with potassium-bearing hydrothermal fluids or sea water.
\end{abstract}

\section{INTRODUCTION}

Volcanic flow rocks were cored at Site 464 on northern Hess Rise and at Site 465 (Hole 465A) on southern Hess Rise (Fig. 1) during DSDP Leg 62 . About $16 \mathrm{~cm}$ of basalt were recovered from Hole 464, and approximately 24 meters of silicic flow rocks were recovered from a 64-meter interval through several flows cored at Site 465 . Whole-rock and rare-earth chemical analyses indicate that the basalt from Hole 464 is tholeiite, a transitional mid-ocean-ridge basalt (MORB), and that the flow rocks from Hole 465A are trachytes analoous to differentiated alkali-basalt magmas of oceanic islands. The basalt is extensively altered, replaced by calcite, smectite, and mixed-layer clay minerals (see Seifert and others, this volume). Isotopic data indicate a hydrothermal origin for the smectites (J. R. O'Neill, pers. comm.). Sea water is also a possible cause of alteration.

Feldspars, along with occasional opaque minerals, are the principal crystalline phases remaining in the altered volcanic flows from Sites 464 and 465 . This paper describes the feldspar compositions of rocks from Holes 464 and $465 \mathrm{~A}$ and relates the compositions to primary magmatic processes and subsequent alteration. Analyses were done on the U.S. Geological Survey ARL EMX-SM electron microprobe in Menlo Park. The operating conditions were $15 \mathrm{kv}$ accelerating voltage, $0.01 \mu \mathrm{mm}$ beam current, and $10 \mu \mathrm{m}$ defocused beam. Natural feldspars and synthetic feldspar glasses were used as standards.

\section{RESULTS}

The most common feldspar crystal habits are laths and microlites; phenocrysts occur in samples from the lowest section of Core 465A-46. Phenocrysts range from 0.25 to $3.0 \mathrm{~mm}$ in maximum dimension; laths and microlites range from 0.05 to $0.3 \mathrm{~mm}$ in length. Simple Carlsbad and albite twinning are prevalent in phenocrysts; only albite twins are evident in laths.

Plagioclase grains from Hole $465 \mathrm{~A}$ commonly have altered, spongy cores partly replaced by potassium feld-

\footnotetext{
${ }^{1}$ Initial Reports of the Deep Sea Drilling Project, Volume 62.
}

Electron-microprobe analyses of the feldspars are plotted in Figure 2 and listed in Table 1. For each sample, groundmass and phenocryst compositions overlap. The four analyses with highest anorthite values $\left(\mathrm{An}_{55-45}\right)$ are from a sample of tholeiite from the bottom of Hole 464. The plagioclase analyses of samples from Hole 465A define a fractionation curve (sketched in figure 2) that reflects slight potassium enrichment paralleling decreasing calcium content $\left(\mathrm{An}_{45-20}\right)$. The extremely high potassium $\left(\mathrm{Or}_{>90}\right)$ analyses represent cores of grains, some patchy areas, and rims of plagioclase grains in the trachytic rocks of Hole 465A.

Analyses that plot off the fractionation trend (with up to $40 \%$ orthoclase) are probably composite analyses of potassium feldspar and plagioclase zones not resolved in the broad defocused beam of the electron microprobe. The resulting analyses reflect various combinations of plagioclase and orthoclase which plot in metastable fields and off trend. Similarly, whole-rock analyses (Seifert and others, this volume) with high potassium produce normative feldspars that also plot in metastable fields.

Except for the presence of more phenocrysts in the bottom sections, there is mineralogical uniformity among the plagioclases in the 64-meter interval sampled in Hole 465A. The feldspars are neither more sodic nor potassic down-core, so no differentiation trend is apparent in the several flows of this sampling interval. Replacement by potassium feldspar in among the Hole 465 A samples is also independent of depth in the core.

\section{DISCUSSION}

Plagioclase $\left(\mathrm{An}_{55-45}\right)$ from the basaltic flow at the bottom of Hole 464 is labradorite, one analysis being slightly more sodic. This composition range is compatible with the tholeiitic basalt host (Carmichael, 1963), but is slightly more sodium-rich than comparable MORB plagioclase (Jackson and others, 1976).

Two generations of feldspars are found in the volcanic flows cored at Site 465A: an earlier, potassiumpoor plagioclase $\left(\mathrm{An}_{45-20}\right)$ that crystallized from the volcanic melt, and a later, nearly pure potassium feld- 


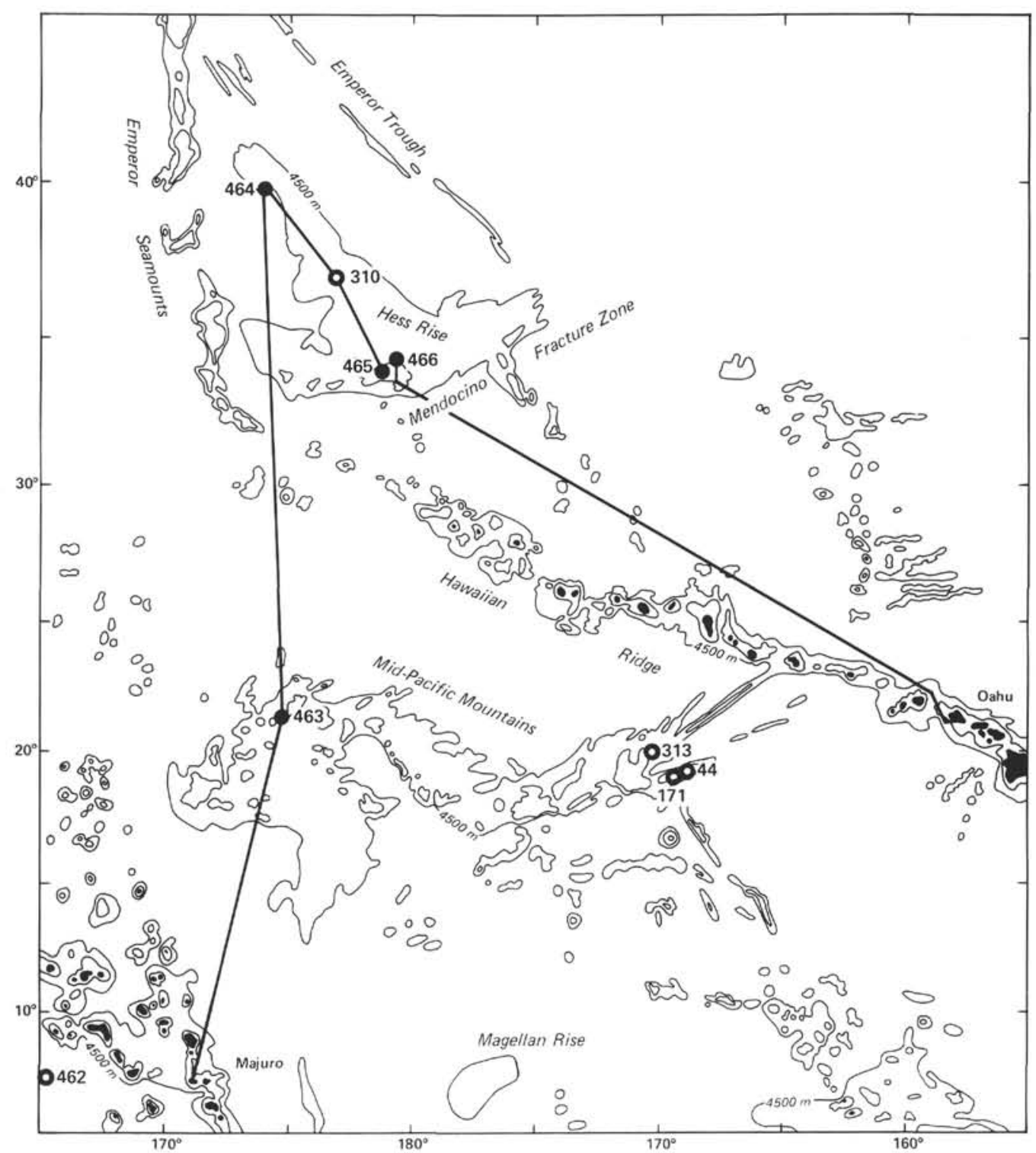

Figure 1. Location of Hess Rise and the DSDP sites drilled during Leg 62.

spar $\left(\mathrm{Or}_{>90}\right)$. Stewart et al. (1973) have concluded that a similar chemical and morphological relationship in plagioclases from DSDP Hole 192 alkali basalt is due to secondary replacement, as opposed to xenocryst accumulation. They also reviewed the work of Carmichael (1963) and Seck (1971), which demonstrates that such potassium-rich feldspar cannot be a coprecipitating phase with plagioclase unless almost pure anorthite is also present.

No structural information on these feldspars is available, but similar alkali feldspars in other altered volcanic rocks have been identified as the disordered hightemperature polymorph sanidine (Stewart and others, 1973).

The potassium feldspars are probably the result of alteration by potassium-bearing hydrothermal fluids (Humphris and Thompson, 1978; Mottl and Holland, 1978) or sea water (Bloch and Bischoff, 1979; Dal- rymple and others, in press). The andesine and oligoclase in lavas of Hole $465 \mathrm{~A}$ are typical of trachytes (Carmichael, 1965), but could also be hosted by less-differentiated hawaiites or mugearites (Keil and others, 1972). Plagioclase data do not define a precise whole-rock composition where that composition is disguised by alteration, as in the case of these rocks.

\section{ACKNOWLEDGMENTS}

I would like to thank David Clague, Randy Koski, and Tracy Vallier for helpful comments and discussions.

\section{REFERENCES}

Block, S., and Bischoff, J. L., 1979. The effect of low-temperature alteration of basalt on the oceanic budget of potassium. Geology, 7:193-196.

Carmichael, I. S. E., 1963. The crystallization of feldspar in volcanic acid glass. Geol. Soc. London Quart. J., 119:95-131.

, 1965. Trachytes and their feldspar phenocrysts. Mineral. Mag., 34:107-125. 
Dalrymple, G. B., Lanphere, M. A., and Natland, J. H., in press. $\mathrm{K}-\mathrm{Ar}$ minimum age for Meiji Guyot, Emperor Seamount Chain. In Jackson, E. D., Koizumi, I., et al., Init. Repts. DSDP, 55: Washington (U.S. Govt. Printing Office).

Humphris, S. E., and Thompson, G., 1978. Hydrothermal alteration of oceanic basalts by seawater. Geochim. Cosmochim. Acta, $4: 107-125$

Jackson, E. D., Bargar, K. E., Fabbi, B. P., et al., C., 1976. Petrology of the basaltic rocks drilled on Leg 33 of the Deep Sea Drilling Project. In Schlanger, S. O., Jackson, E. D., et al., Init. Repts. DSDP, 33: Washington (U.S. Govt. Printing Office), 571-630.

Keil, K., Fodor, R. V., and Bunch, T. E., 1972. Contributions to the mineral chemistry of Hawaiian rocks. II. Feldspars and interstitial material in rocks from Haleakala and West Maui volcanoes, Maui, Hawaii. Contr. Mineral. Petrol., 37:253-276.

Mottl, M. J., and Holland, H. D., 1978. Chemical exchange during hydrothermal alteration of basalt by seawater. 1. Experimental results for major and minor components of seawater. Geochim. Cosmochim. Acta, 42:1103-1115.

Seck, H. A., 1971. Der Einfluss des Drucks auf die Zusammensetzung koexistierender Alkalifeldspate und Plagioklase im System NaAl $\mathrm{Si}_{3} \mathrm{O}_{8}-\mathrm{KAlSi}_{3}-\mathrm{CaAl}_{2} \mathrm{Si}_{2} \mathrm{O}_{8}-\mathrm{H}_{2} \mathrm{O}$. Contr. Mineral. Petrol., 31: 67-86.

Stewart, R. J., Natland, J. H., and Glassley, W. R., 1973. Petrology of yolcanic rocks recovered on DSDP Leg 19 from the north Pacific Ocean and the Bering Sea. In Creager, J. S., Scholl. D. W., et al., Init. Repts. DSDP, 19: Washington (U.S. Govt. Printing Office), 615-627.

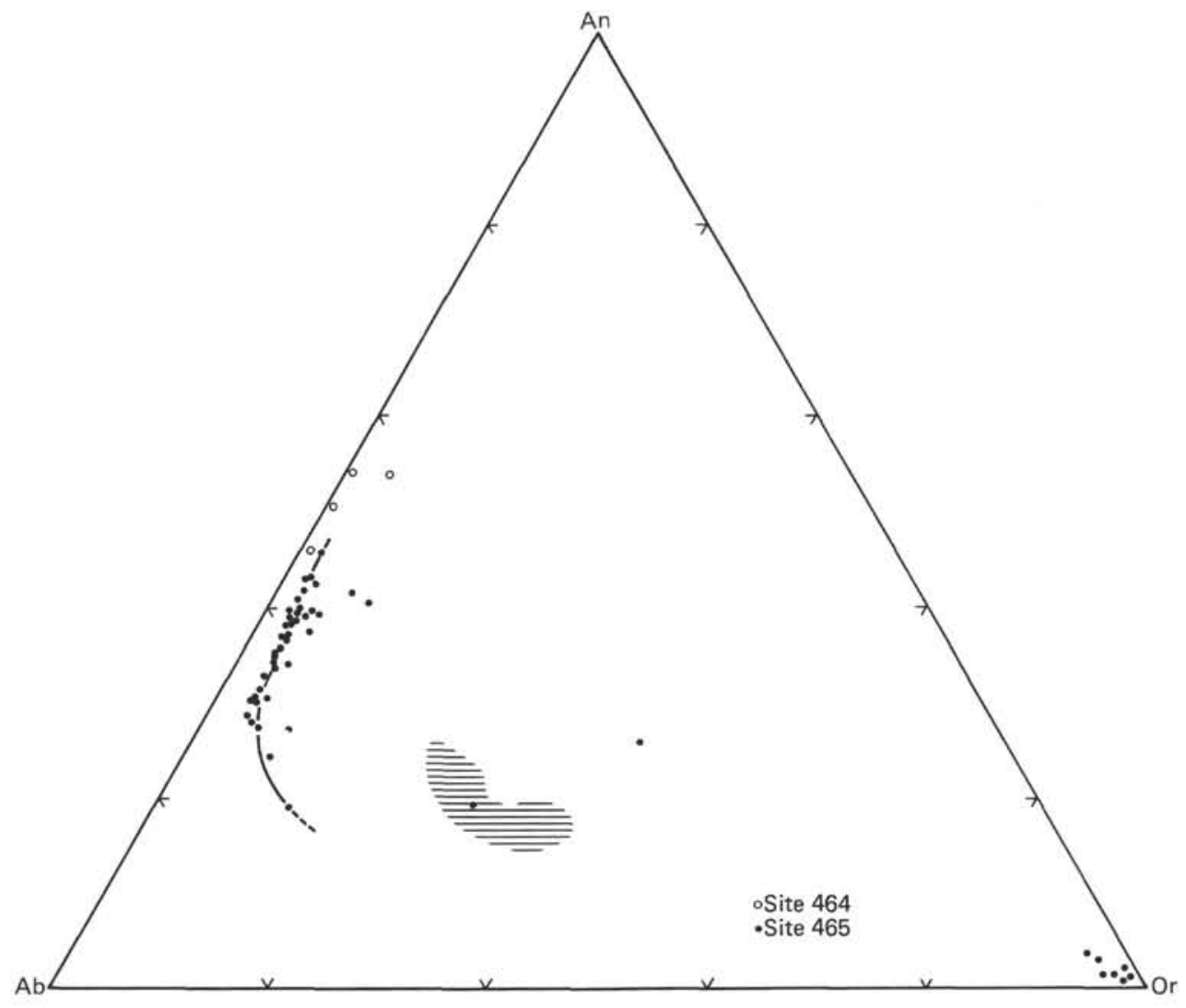

Figure 2. Ternary molecular An-Ab-Or plot of feldspars from DSDP Sites 464 and 465 on Hess Rise. Normative-feldspar data (hachured area) are from Seifert et al. (this volume). 
Table 1. Electron-microprobe analyses of feldspars from Hess Rise.

\begin{tabular}{|c|c|c|c|c|c|c|c|c|c|c|c|c|c|c|c|}
\hline \multirow[b]{2}{*}{ Sample } & & \multirow[b]{2}{*}{$\mathrm{SiO}_{2}$} & \multirow[b]{2}{*}{$\mathrm{Al}_{2} \mathrm{O}_{3}$} & \multirow[b]{2}{*}{$\mathrm{CaO}$} & \multirow[b]{2}{*}{$\mathrm{Na}_{2} \mathrm{O}$} & \multirow[b]{2}{*}{$\mathrm{K}_{2} \mathrm{O}$} & \multirow[b]{2}{*}{ Total } & \multicolumn{5}{|c|}{ Cations/32 Oxygens } & \multicolumn{3}{|c|}{ Molecular Ratios } \\
\hline & & & & & & & & $\mathrm{Si}$ & Al & $\mathrm{Ca}$ & $\mathrm{Na}$ & $\mathrm{K}$ & Or & $\mathrm{Ab}$ & An \\
\hline $464-34, C C, 21 \mathrm{~cm}$ & & $\begin{array}{l}55.92 \\
54.61 \\
54.61 \\
54.80\end{array}$ & $\begin{array}{l}26.58 \\
27.23 \\
27.29 \\
27.69\end{array}$ & $\begin{array}{r}9.45 \\
10.49 \\
11.05 \\
11.17\end{array}$ & $\begin{array}{l}6.03 \\
5.57 \\
4.74 \\
5.17\end{array}$ & $\begin{array}{l}0.20 \\
0.09 \\
0.70 \\
0.10\end{array}$ & $\begin{array}{l}98.17 \\
98.00 \\
98.39 \\
98.93\end{array}$ & $\begin{array}{r}10.231 \\
10.041 \\
10.024 \\
9.987\end{array}$ & $\begin{array}{l}5.730 \\
5.900 \\
5.903 \\
5.947\end{array}$ & $\begin{array}{l}1.852 \\
2.067 \\
2.173 \\
2.181\end{array}$ & $\begin{array}{l}2.138 \\
1.984 \\
1.687 \\
1.826\end{array}$ & $\begin{array}{l}0.046 \\
0.021 \\
0.163 \\
0.023\end{array}$ & $\begin{array}{l}1.1 \\
0.5 \\
4.1 \\
0.6\end{array}$ & $\begin{array}{l}53.0 \\
48.7 \\
41.9 \\
45.3\end{array}$ & $\begin{array}{l}45.9 \\
50.8 \\
54.0 \\
54.1\end{array}$ \\
\hline $465 \mathrm{~A}-40-3,31$ & & $\begin{array}{l}56.59 \\
58.43 \\
58.91 \\
59.99 \\
60.68 \\
59.39\end{array}$ & $\begin{array}{l}26.17 \\
25.17 \\
25.10 \\
23.43 \\
23.77 \\
24.35\end{array}$ & $\begin{array}{l}9.59 \\
8.11 \\
7.98 \\
6.53 \\
5.85 \\
7.09\end{array}$ & $\begin{array}{l}6.09 \\
6.79 \\
6.87 \\
7.46 \\
7.77 \\
6.88\end{array}$ & $\begin{array}{l}0.33 \\
0.45 \\
0.46 \\
0.61 \\
0.78 \\
0.50\end{array}$ & $\begin{array}{l}98.77 \\
98.95 \\
99.32 \\
98.02 \\
98.85 \\
98.22\end{array}$ & $\begin{array}{l}10.299 \\
10.569 \\
10.609 \\
10.907 \\
10.929 \\
10.773\end{array}$ & $\begin{array}{l}5.613 \\
5.366 \\
5.328 \\
5.020 \\
5.046 \\
5.204\end{array}$ & $\begin{array}{l}1.871 \\
1.572 \\
1.539 \\
1.271 \\
1.128 \\
1.378\end{array}$ & $\begin{array}{l}2.148 \\
2.380 \\
2.398 \\
2.628 \\
2.715 \\
2.421\end{array}$ & $\begin{array}{l}0.076 \\
0.103 \\
0.106 \\
0.142 \\
0.179 \\
0.116\end{array}$ & $\begin{array}{l}1.9 \\
2.5 \\
2.6 \\
3.5 \\
4.4 \\
3.0\end{array}$ & $\begin{array}{l}52.5 \\
58.7 \\
59.3 \\
65.0 \\
67.5 \\
61.8\end{array}$ & $\begin{array}{l}45.7 \\
38.8 \\
38.1 \\
31.5 \\
28.1 \\
35.2\end{array}$ \\
\hline $465 \mathrm{~A}-41-2,82$ & $\begin{array}{l}\operatorname{rim}^{1} \\
\text { core }^{1} \\
\text { rim }^{2} \\
\text { core }^{2} \\
\text { rim }^{3} \\
\text { core }^{3}\end{array}$ & $\begin{array}{l}60.83 \\
60.70 \\
60.57 \\
58.59 \\
63.74 \\
64.30 \\
65.31\end{array}$ & $\begin{array}{l}24.00 \\
25.03 \\
24.38 \\
24.75 \\
18.45 \\
19.06 \\
18.48\end{array}$ & $\begin{array}{l}5.88 \\
6.26 \\
6.24 \\
7.18 \\
0.31 \\
0.63 \\
0.20\end{array}$ & $\begin{array}{l}7.65 \\
7.39 \\
7.44 \\
6.82 \\
0.34 \\
0.31 \\
0.13\end{array}$ & $\begin{array}{r}0.67 \\
0.60 \\
0.62 \\
0.58 \\
15.18 \\
15.47 \\
14.56\end{array}$ & $\begin{array}{l}99.03 \\
99.98 \\
99.25 \\
97.93 \\
98.02 \\
99.77 \\
98.68\end{array}$ & $\begin{array}{l}10.922 \\
10.797 \\
10.856 \\
10.677 \\
11.967 \\
11.884 \\
12.087\end{array}$ & $\begin{array}{l}5.078 \\
5.248 \\
5.150 \\
5.315 \\
4.083 \\
4.152 \\
4.030\end{array}$ & $\begin{array}{l}1.131 \\
1.192 \\
1.199 \\
1.402 \\
0.063 \\
0.126 \\
0.039\end{array}$ & $\begin{array}{l}2.661 \\
2.549 \\
2.587 \\
2.409 \\
0.123 \\
0.110 \\
0.047\end{array}$ & $\begin{array}{l}0.153 \\
0.136 \\
0.141 \\
0.136 \\
3.636 \\
3.648 \\
3.437\end{array}$ & $\begin{array}{r}3.9 \\
3.5 \\
3.6 \\
3.4 \\
95.1 \\
93.9 \\
97.6\end{array}$ & $\begin{array}{r}67.5 \\
65.7 \\
65.9 \\
61.0 \\
3.2 \\
2.8 \\
1.3\end{array}$ & $\begin{array}{r}28.7 \\
30.8 \\
30.5 \\
35.5 \\
1.6 \\
3.2 \\
1.1\end{array}$ \\
\hline $465 \mathrm{~A}-41-2,137$ & & $\begin{array}{l}57.84 \\
64.96 \\
59.00 \\
58.45 \\
58.15\end{array}$ & $\begin{array}{l}26.65 \\
18.51 \\
25.22 \\
26.74 \\
25.25\end{array}$ & $\begin{array}{l}8.81 \\
0.23 \\
7.10 \\
8.02 \\
7.43\end{array}$ & $\begin{array}{l}6.23 \\
0.14 \\
7.10 \\
6.53 \\
6.63\end{array}$ & $\begin{array}{r}0.41 \\
15.60 \\
0.59 \\
0.40 \\
0.46\end{array}$ & $\begin{array}{r}99.95 \\
99.44 \\
99.01 \\
100.15 \\
97.93\end{array}$ & $\begin{array}{l}10.367 \\
12.018 \\
10.640 \\
10.429 \\
10.596\end{array}$ & $\begin{array}{l}5.629 \\
4.035 \\
5.360 \\
5.623 \\
5.423\end{array}$ & $\begin{array}{l}1.692 \\
0.045 \\
1.372 \\
1.532 \\
1.451\end{array}$ & $\begin{array}{l}2.166 \\
0.051 \\
2.482 \\
2.258 \\
2.341\end{array}$ & $\begin{array}{l}0.094 \\
3.682 \\
0.136 \\
0.092 \\
0.106\end{array}$ & $\begin{array}{r}2.4 \\
97.5 \\
3.4 \\
2.4 \\
2.7\end{array}$ & $\begin{array}{r}54.8 \\
1.3 \\
62.2 \\
58.2 \\
60.0\end{array}$ & $\begin{array}{r}42.8 \\
1.2 \\
34.4 \\
39.5 \\
37.2\end{array}$ \\
\hline $465 \mathrm{~A}-42-1,123$ & & $\begin{array}{l}57.24 \\
59.86 \\
59.92 \\
59.03\end{array}$ & $\begin{array}{l}27.04 \\
24.16 \\
24.72 \\
25.62\end{array}$ & $\begin{array}{l}8.62 \\
6.19 \\
6.80 \\
7.80\end{array}$ & $\begin{array}{l}6.17 \\
7.48 \\
7.02 \\
6.49\end{array}$ & $\begin{array}{l}0.34 \\
0.64 \\
0.64 \\
0.43\end{array}$ & $\begin{array}{l}99.40 \\
98.34 \\
99.10 \\
99.37\end{array}$ & $\begin{array}{l}10.308 \\
10.839 \\
10.770 \\
10.596\end{array}$ & $\begin{array}{l}5.738 \\
5.156 \\
5.236 \\
5.420\end{array}$ & $\begin{array}{l}1.662 \\
1.201\end{array}$ & $\begin{array}{l}2.155 \\
2.626 \\
2.448 \\
2.258\end{array}$ & & $\begin{array}{l}2.0 \\
3.7 \\
3.8 \\
2.5\end{array}$ & $\begin{array}{l}55.3 \\
66.1 \\
62.7 \\
58.6\end{array}$ & $\begin{array}{l}42.7 \\
30.2 \\
33.5 \\
38.9\end{array}$ \\
\hline $465 \mathrm{~A}-42-3,84$ & & $\begin{array}{l}57.75 \\
64.57 \\
57.64 \\
59.64\end{array}$ & $\begin{array}{l}26.06 \\
18.31 \\
25.28 \\
24.72\end{array}$ & $\begin{array}{l}7.89 \\
0.43 \\
7.83 \\
7.13\end{array}$ & & $\begin{array}{r}0.44 \\
16.05 \\
0.46 \\
0.53\end{array}$ & $\begin{array}{l}98.68 \\
99.49 \\
97.81 \\
99.08\end{array}$ & $\begin{array}{l}10.461 \\
11.990 \\
10.537 \\
10.734\end{array}$ & $\begin{array}{l}5.564 \\
4.007 \\
5.446 \\
5.243\end{array}$ & $\begin{array}{l}1.531 \\
0.086 \\
1.534 \\
1.376\end{array}$ & $\begin{array}{l}2.300 \\
0.046 \\
2.338 \\
2.463\end{array}$ & $\begin{array}{l}0.101 \\
3.801 \\
0.107 \\
0.122\end{array}$ & $\begin{array}{r}2.6 \\
96.6 \\
2.7 \\
3.1\end{array}$ & $\begin{array}{r}58.5 \\
1.2 \\
58.8 \\
62.2\end{array}$ & $\begin{array}{r}38.9 \\
2.2 \\
38.5 \\
34.7\end{array}$ \\
\hline $465 \mathrm{~A}-43-1,64$ & $\begin{array}{l}\text { rim } \\
\text { core }\end{array}$ & $\begin{array}{l}64.26 \\
62.07 \\
57.53 \\
58.47 \\
58.11 \\
63.06 \\
57.17\end{array}$ & $\begin{array}{l}18.41 \\
23.94 \\
26.19 \\
25.59 \\
25.36 \\
19.11 \\
26.23\end{array}$ & $\begin{array}{l}0.74 \\
5.06 \\
8.85 \\
7.74 \\
7.65 \\
0.23 \\
8.27\end{array}$ & $\begin{array}{l}0.43 \\
3.56 \\
6.29 \\
6.93 \\
6.51 \\
0.18 \\
6.40\end{array}$ & $\begin{array}{r}15.59 \\
6.69 \\
0.53 \\
0.46 \\
0.86 \\
16.53 \\
0.43\end{array}$ & $\begin{array}{r}99.42 \\
101.31 \\
99.39 \\
99.19 \\
98.48 \\
99.10 \\
98.49\end{array}$ & $\begin{array}{l}11.811 \\
10.393\end{array}$ & $\begin{array}{l}5.431 \\
4.217 \\
5.619\end{array}$ & $\begin{array}{l}1.489 \\
0.046 \\
1.610\end{array}$ & $\begin{array}{l}0.154 \\
1.229 \\
2.202 \\
2.423 \\
2.293 \\
0.065 \\
2.254\end{array}$ & $\begin{array}{l}3.950 \\
0.099\end{array}$ & $\begin{array}{r}92.5 \\
40.9 \\
3.0 \\
2.7 \\
5.0 \\
97.3 \\
2.5\end{array}$ & $\begin{array}{r}3.9 \\
33.1 \\
54.6 \\
60.2 \\
57.6 \\
1.6 \\
56.9\end{array}$ & $\begin{array}{r}3.7 \\
26.0 \\
42.4 \\
37.1 \\
37.4 \\
1.1 \\
40.6\end{array}$ \\
\hline $465 \mathrm{~A}-43-2,107$ & & $\begin{array}{l}57.10 \\
56.80 \\
58.03 \\
60.40\end{array}$ & $\begin{array}{l}25.25 \\
26.25 \\
26.54 \\
23.52\end{array}$ & $\begin{array}{l}8.02 \\
8.61 \\
8.11 \\
5.76\end{array}$ & $\begin{array}{l}6.47 \\
6.38 \\
6.50 \\
7.72\end{array}$ & $\begin{array}{l}0.67 \\
0.44 \\
0.51 \\
0.91\end{array}$ & & $\begin{array}{l}10.416 \\
10.944\end{array}$ & & $\begin{array}{l}1.560 \\
1.119\end{array}$ & $\begin{array}{l}2.260 \\
2.712\end{array}$ & & $\begin{array}{l}3.0 \\
5.2\end{array}$ & $\begin{array}{l}57.0 \\
55.9 \\
57.4 \\
67.1\end{array}$ & $\begin{array}{l}39.1 \\
41.6 \\
39.6 \\
27.7\end{array}$ \\
\hline $465 \mathrm{~A}-44-2,37$ & $\begin{array}{l}\operatorname{rim}^{1} \\
\text { core } \\
\text { rim }^{2} \\
\text { core }^{2}\end{array}$ & $\begin{array}{l}60.55 \\
59.73 \\
65.17 \\
60.67 \\
60.48 \\
58.36 \\
58.27\end{array}$ & $\begin{array}{l}25.21 \\
24.96 \\
22.32 \\
23.06 \\
23.15 \\
25.55 \\
24.68\end{array}$ & $\begin{array}{l}3.03 \\
5.07 \\
5.65 \\
7.54 \\
7.85\end{array}$ & $\begin{array}{l}6.99 \\
6.97 \\
5.31 \\
7.77 \\
7.41 \\
6.83 \\
6.69\end{array}$ & $\begin{array}{l}0.62 \\
0.79 \\
4.45 \\
1.41 \\
1.45 \\
0.58 \\
0.52\end{array}$ & $\begin{array}{r}100.30 \\
99.44 \\
100.88 \\
97.99 \\
98.15 \\
98.86 \\
98.00\end{array}$ & $\begin{array}{l}11.480 \\
11.032 \\
10.993\end{array}$ & $\begin{array}{l}4.633 \\
4.942 \\
4.958\end{array}$ & $\begin{array}{l}0.686 \\
0.988 \\
1.101 \\
1.461 \\
1.534\end{array}$ & $\begin{array}{l}1.812 \\
2.741 \\
2.612\end{array}$ & $\begin{array}{l}0.141 \\
0.181 \\
1.000 \\
0.328\end{array}$ & $\begin{array}{r}28.6 \\
8.1 \\
8.3 \\
3.3 \\
3.0\end{array}$ & $\begin{array}{l}62.3 \\
61.4 \\
51.8 \\
67.6 \\
64.5 \\
60.0 \\
58.8\end{array}$ & $\begin{array}{l}34.0 \\
34.0 \\
19.6 \\
24.3 \\
27.2 \\
36.6 \\
38.2\end{array}$ \\
\hline $165 \mathrm{~A}-46-1,37$ & $\begin{array}{l}\operatorname{rim}^{1} \\
\text { core }^{1} \\
\text { rim }^{2} \\
\text { core }^{2} \\
\text { rim }^{3} \\
\text { core }^{3}\end{array}$ & $\begin{array}{l}59.69 \\
58.40 \\
57.31 \\
58.13 \\
58.06 \\
63.31 \\
62.63\end{array}$ & $\begin{array}{l}24.94 \\
26.46 \\
26.72 \\
26.23 \\
25.56 \\
18.42 \\
21.79\end{array}$ & $\begin{array}{l}6.48 \\
7.89 \\
8.54 \\
8.25 \\
8.11 \\
0.31 \\
3.97\end{array}$ & $\begin{array}{l}7.58 \\
6.66 \\
5.89 \\
6.73 \\
6.33 \\
0.25 \\
7.81\end{array}$ & $\begin{array}{r}0.82 \\
0.55 \\
1.19 \\
0.38 \\
0.85 \\
15.53 \\
2.12\end{array}$ & $\begin{array}{l}99.50 \\
99.96 \\
99.65 \\
99.72 \\
98.91 \\
97.83 \\
98.31\end{array}$ & $\begin{array}{l}10.516 \\
11.940 \\
11.326\end{array}$ & $\begin{array}{l}5.550 \\
5.456 \\
4.095 \\
4.644\end{array}$ & $\begin{array}{l}1.245 \\
1.513 \\
1.649 \\
1.587 \\
1.574 \\
0.063 \\
0.769\end{array}$ & $\begin{array}{l}2.222 \\
0.092 \\
2.738\end{array}$ & & $\begin{array}{l}4.6 \\
3.2 \\
6.8 \\
2.2 \\
4.9 \\
96.0 \\
12.2\end{array}$ & $\begin{array}{r}64.8 \\
58.5 \\
51.7 \\
58.3 \\
55.7 \\
2.4 \\
68.5\end{array}$ & $\begin{array}{r}30.6 \\
38.3 \\
41.4 \\
39.5 \\
39.4 \\
1.6 \\
19.3\end{array}$ \\
\hline $465 \mathrm{~A}-46-2$ & $\begin{array}{l}\text { zone }^{1} \\
\text { zone }^{2}\end{array}$ & & & & $\begin{array}{l}6.92 \\
6.36 \\
7.33\end{array}$ & $\begin{array}{l}0.51 \\
0.43 \\
0.57\end{array}$ & $\begin{array}{l}99.98 \\
98.08 \\
99.97\end{array}$ & $\begin{array}{l}10.648 \\
10.384 \\
10.757\end{array}$ & $\begin{array}{l}5.309 \\
5.583 \\
5.233\end{array}$ & $\begin{array}{l}1.485 \\
1.680 \\
1.304\end{array}$ & $\begin{array}{l}2.396 \\
2.253 \\
2.534\end{array}$ & & $\begin{array}{l}2.9 \\
2.5 \\
3.3\end{array}$ & $\begin{array}{l}60.0 \\
55.9 \\
63.9\end{array}$ & $\begin{array}{l}37.1 \\
41.6 \\
32.9\end{array}$ \\
\hline 16 & $\begin{array}{l}\text { zone }^{1} \\
\text { zone }^{2}\end{array}$ & $\begin{array}{l}59.90 \\
59.42 \\
57.92 \\
59.45 \\
58.55 \\
59.17\end{array}$ & 26.04 & & $\begin{array}{l}6.96 \\
7.63 \\
6.39 \\
7.11 \\
5.77 \\
6.55\end{array}$ & $\begin{array}{l}0.53 \\
0.58\end{array}$ & $\begin{array}{r}101.24 \\
98.84 \\
99.21 \\
99.36 \\
99.38 \\
101.11\end{array}$ & $\begin{array}{l}10.573 \\
10.721\end{array}$ & $\begin{array}{l}5.416 \\
5.286 \\
5.550 \\
5.297 \\
5.357 \\
5.575\end{array}$ & $\begin{array}{l}1.478 \\
1.226 \\
1.565\end{array}$ & $\begin{array}{l}2.383 \\
2.670 \\
2.235\end{array}$ & 0.117 & $\begin{array}{l}3.0 \\
3.3 \\
4.1 \\
3.2 \\
9.0 \\
3.0\end{array}$ & $\begin{array}{l}59.9 \\
66.2 \\
56.4 \\
62.0 \\
50.6 \\
57.6\end{array}$ & $\begin{array}{l}37.1 \\
30.4 \\
39.5 \\
34.9 \\
40.4 \\
39.4\end{array}$ \\
\hline
\end{tabular}

Note: Rim, core, and zone designations refer to a particular grain. Unlabeled analyses are whole-grain averages. 